Mykychak Y., cardiovascular surgeon, Cardiac surgery department, https://orcid.org/0000-0002-7712-7275

Kozhokar D., pediatric surgery resident, Cardiac surgery department, https://orcid.org/0000-0002-3579-8732

Yusifli I., pediatric surgery resident, Cardiac surgery department, https://orcid.org/0000-0002-6787-6897

Morkovkina A., radiologist, Radiology department

Tammo R., PhD, radiologist, Radiology department, https://orcid.org/0000-0001-7506-9449

Yemets I., PhD., professor, cardiovascular surgeon, https://orcid.org/0000-0002-1893-0164

Ukrainian Children's Cardiac Center, Kyiv, Ukraine

\title{
Comparison of Echocardiography and Computed Tomography/Magnetic Resonance Imaging for Assessing of Pulmonary Vein Stenosis in Children
}

\begin{abstract}
Pulmonary vein (PV) stenosis is a rare disease with high recurrence and mortality rates.
Objective. This study was aimed to evaluate the diagnostic accuracy of echocardiography versus cross-sectional modalities.

Material and methods. Our study includes 11 consecutive patients who underwent a comprehensive PV anatomy assessment between April 2018 and June 2019 at Ukrainian Children's Cardiac Center, Kyiv. Echocardiographic studies of each pulmonary vein followed by computed tomography (CT) or magnetic resonance imaging (MRI) were performed in all patients. A total of 27 CT/MRI studies were performed.

Results. There were four patients whose echocardiography findings were completely confirmed by CT/MRI method. In some patients significant stenosis was underestimated by echocardiography. Sensitivity and specificity were 86.4 and 88.9 for echo, as well as 100 and 94.4 for CT/MRI, respectively.

Conclusion. This study recognizes the limitations of echocardiography as an imaging tool for pulmonary veins morphology assessment. Cross-sectional studies overcome these limitations and provide excellent morphological as well as functional (MRI) evaluation of pulmonary veins.
\end{abstract}

Keywords: pulmonary vein stenosis, echocardiography, computed tomography, magnetic resonance imaging.

\section{Introduction}

Pulmonary vein stenosis (PVS) in children is a rare but serious condition $[1,2]$ which may occur in $10-17 \%$ of patients after total anomalous pulmonary venous connection (TAPVC) repair $[3,4]$, or presents as a congenital/primary disease bearing high recurrence and mortality rates of $46-80 \%$ [5]. Pulmonary veins imaging is important for preoperative planning, outcomes assessment and followup. Different imaging modalities can provide valuable information on severity and location of the lesions. However, each method has its own benefits and limitations.

In this study we aimed to compare the diagnostic accuracy of echocardiography versus cross-sectional modalities (computed tomography or magnetic resonance imaging) for assessment of pulmonary veins characteristics and to develop an institutional PVS detection and surveillance algorithm.

\section{Materials and methods}

Our study includes 11 consecutive patients (Table 1) who underwent a comprehensive PV anatomy assessment between April 2018 and June 2019 at Ukrainian Children's
Cardiac Center, Kyiv. Two-dimensional and Doppler echocardiographic studies of each pulmonary vein with following computed tomography (CT) or magnetic resonance imaging (MRI) were performed in all patients. A total of 27 CT/MRI studies were performed, and each was compared to the respective echocardiographic study.

Echocardiography. Each PV was assessed by echocardiography according to a standardized imaging protocol described by Kalfa et al. [6], consisting of three components: (1) severity of disease measured by mean echocardiographic pressure gradient and (2) extent of stenosis in each individual pulmonary vein (PV) and (3) uni- or bilateral extent of the disease. ECHSA Score was used to summarize the findings. A mean echo gradient of $<2 \mathrm{mmHg}$, biphasic blood flow and the absence of PV narrowing confirmed the absence of stenosis. A mean echo gradient of 2 to $4 \mathrm{mmHg}, 5$ to $7 \mathrm{mmHg}$ and $>7 \mathrm{mmHg}$ indicated mild, moderate and severe stenosis, respectively. Involvement of only a short length of the PV and presence of upstream dilatation was defined as a focal stenosis. Stenosis was considered diffuse if a significant length of the PV was af- 
fected with no upstream dilation of the PV. Each of PV has been assigned a score of 0-4: 0 for no stenosis; 1, 2, 3 for discrete mild, moderate and severe stenosis; $1.5,2.5$, and 3.5 for diffuse stenosis; 4 for atresia or occlusion.

All individual pulmonary veins scores were summarized to calculate the total score. If bilateral disease was present, 2 points have been added. Possible score range was $0-18$ [14].

Cross-sectional imaging modalities. For CT/MRI assessment of pulmonary veins, we used the method proposed by the Toronto group [7, 10]. Short and long cross-sectional diameters of each PV at the left atrial junction (downstream) and the bifurcation (upstream), the individual PV cross-sectional area indexed to body surface area (PVCSAi) [8] and total PV cross-sectional area indexed to body surface area (TCSAi) were measured. In addition, in patients undergoing MRI pulmonary artery flow redistribution was assessed.

Statistical analysis. Predictive accuracy for echocardiography and cross-sectional modalities was assessed by calculating the area under the receiver operating characteristics (ROC) curve.

MedCalc Statistical Software version 18.2.1 (MedCalc Software bvba, Ostend, Belgium) was used for statistical analyses.

\section{Results}

The results of echocardiography findings and crosssectional studies compared with intraoperative findings are presented in Table 1.

Echocardiography findings in patients 2, 6, 7 and 10 were completely confirmed by CT/MRI method.

In patient $1 \mathrm{CT}$ scan revealed left upper PV atresia and left lower PV severe stenosis, whereas echocardiography showed only moderate stenosis of the left lower PV.

In patient 11 echocardiography (Fig. 1) which was performed in a month after primary echo study revealed decreased mean echo gradients on left pulmonary veins.

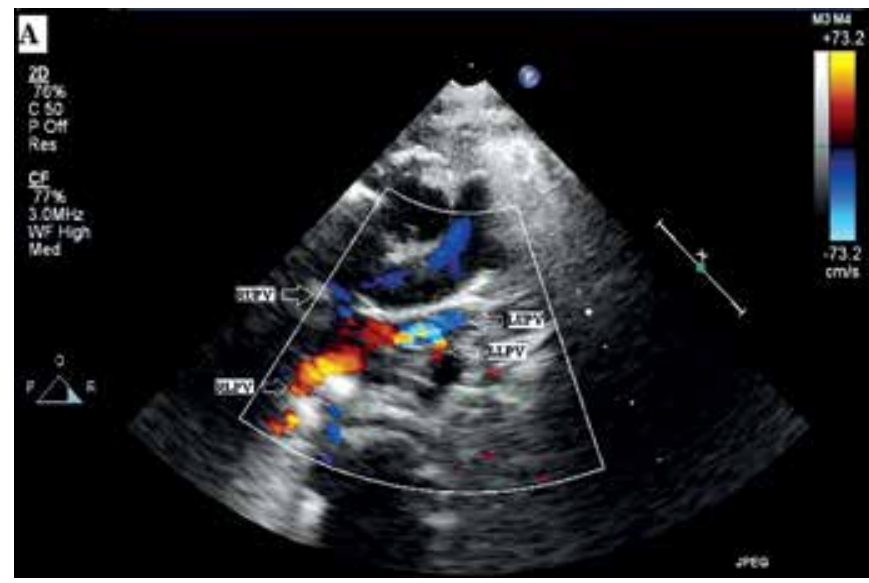

Figure 1. Echocardiography of the patient with suspected pulmonary vein stenosis

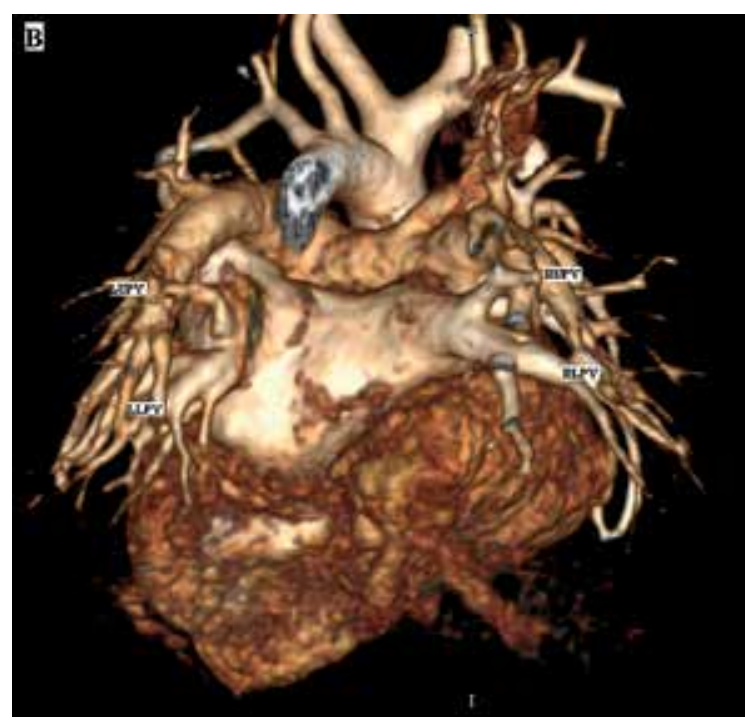

Figure 2. 3D reconstruction image obtained for the same patient. RUPV, right upper pulmonary vein; $R L P V$, right lower $P V$; LUPV, left upper PV; LLPV, left lower PV

Meanwhile, CT (Fig. 2) showed worsening of the stenosis compared to CT imaging performed month earlier. CT scans in the first and third months following sutureless repair on the left pulmonary veins revealed unobstructed flow throw these veins.

Eight months after primary surgery, this patient was readmitted with clinical signs of infective endocarditis. Echocardiography revealed vegetations on tricuspid valve and suspected left pulmonary veins restenosis. CT imaging which was performed to confirm the diagnosis revealed new right upper PV stenosis and left pulmonary veins restenosis.

In patient 8 , in addition to detected secundum atrial septal defect (ASD), severe right upper pulmonary vein (RUPV) stenosis was suspected during echocardiography. Preoperative CT revealed normal dimensions of RUPV. Thus, this patient underwent minimally invasive right midaxillary thoracotomy for ASD closure without unnecessary RUPV intervention.

Patient 9 was admitted to our clinic with signs of pulmonary edema. Echocardiography revealed severe pulmonary hypertension with unclear cause and without relevant pressure gradients over pulmonary veins. Subsequent CT scan showed severe hypoplasia of all pulmonary veins.

All aforementioned patients had primary PVS. Patient 10 was the only one in this series with postsurgical PVS whose CT scan identified stenosis of pulmonary venous collector.

Statistical analysis. Both methods were compared using ROC analysis. ROC curves showed that cross-sectional study had higher sensitivity and specificity (Fig. 3). Thus, 
Table 1

Pulmonary veins characteristics

\begin{tabular}{|c|c|c|c|c|c|c|c|}
\hline Patient & $\begin{array}{l}\text { Baseline Echo } \\
\Delta p m e a n, \mathrm{~mm} \mathrm{Hg}\end{array}$ & $\begin{array}{l}\text { Baseline cross-sectional study, } \\
\text { indexed area of individual vein }\end{array}$ & Intraoperative findings & $\begin{array}{l}\text { Postoperative echo, } \\
\Delta \rho \text { mean, } \mathrm{mm} \mathrm{Hg}\end{array}$ & $\begin{array}{l}\text { Postoperative cross-sectional study, } \\
\text { indexed area of individual vein }\end{array}$ & $\begin{array}{l}\text { Follow-up } \\
\text { Echo, } \\
\text { هpmean, } \mathrm{mm} \\
\mathrm{Hg}\end{array}$ & $\begin{array}{l}\text { Follow-up } \\
\text { cross-sectional } \\
\text { study }\end{array}$ \\
\hline 1 & LUPV - 7 & $\begin{array}{l}\text { LUPV: d:ia - 8.8; LUPV: uia - } 22 \\
\text { LLPV: d:ia - } 26.4\end{array}$ & $\begin{array}{l}\text { LUPV subocclussion, } \\
\text { diffuse } \\
\text { LLPV local stenosis }\end{array}$ & LLPV - 2.1 & $\begin{array}{l}\text { LUPV: d:ia - 12; LUPV: u:ia - 27 } \\
\text { LLPV:d:ia - 25; LLPV: u:ia - } 38\end{array}$ & LLPV -2.1 & $\begin{array}{l}\text { LUPV: d:ia - 12; } \\
\text { LUPV: u:ia - 27 } \\
\text { LLPV: d:ia - 25; } \\
\text { LLPV:u:ia -38 }\end{array}$ \\
\hline 2 & LUPV/LLPV collector - 5 & $\begin{array}{l}\text { LUPV/LLPV collector: d:ia: } 0,15 \\
\text { LUPV/LLPV collector: u:ia: } 3\end{array}$ & $\begin{array}{l}\text { LUPV/LLPV collector } \\
\text { stenosis }\end{array}$ & absence of stenosis & $\mathrm{N}$ cross-sectional area index & LLPV -3 & $\begin{array}{l}N \text { cross-sectional } \\
\text { area index }\end{array}$ \\
\hline 3 & $\begin{array}{l}\text { RUPV - } 5 \\
\text { left PVs weren't visualize }\end{array}$ & $\begin{array}{l}\text { LUPV: d:ia - 10; LUPV:u:ia - } 19 \\
\text { LLPV: d:ia - } 4 \text { LUPV:u:ia - } 25\end{array}$ & $\begin{array}{l}\text { LUPV subocclusion } \\
\text { LLPV occlusion }\end{array}$ & $\begin{array}{l}\text { LUPV }-4 \\
\text { LLPV }-9\end{array}$ & $\begin{array}{l}\text { LUPV: d:ia - 11; LUPV: u:ia - } 20 \\
\text { LLPV:d:ia - } 6 \text { LUPV:u:ia - } 23\end{array}$ & $\begin{array}{l}\text { LUPV }-4 \\
\text { LLPV }-9\end{array}$ & $\begin{array}{l}\text { LUPV:d:ia - 11; } \\
\text { LUPV:u:ia - 20 } \\
\text { LLPV:d:ia - } 6 \\
\text { LUPV:u:ia - } 23\end{array}$ \\
\hline 4 & $\begin{array}{l}\text { RUPV }-5 \\
\text { LLPV }-4\end{array}$ & $\begin{array}{l}\text { LUPV: d:ia - 43; } \\
\text { LLPV:d:ia - } 19\end{array}$ & $\begin{array}{l}\text { RUPV free of stenosis } \\
\text { left PVs local stenosis }\end{array}$ & $\begin{array}{l}\text { RUPV - } 2 \\
\text { LLPV -1 }\end{array}$ & $\begin{array}{l}\text { LUPV: d:ia - 26; LUPV: u:ia - } 30 \\
\text { LLPV: d:ia - } 34\end{array}$ & $\begin{array}{l}\text { RUPV-2 } \\
\text { LLPV-1 }\end{array}$ & $\begin{array}{l}\text { LUPV:d:ia - 26; } \\
\text { LUPV:u:ia - } 30 \\
\text { LLPV: d:ia - } 34\end{array}$ \\
\hline 5 & $\begin{array}{l}\text { RLPV-5 } \\
\text { LLPV-2 } \\
\text { RUPV, LUPV weren't } \\
\text { visualize }\end{array}$ & $\begin{array}{l}\text { RUPV occlusion } \\
\text { LUPV: d:ia - 43; } \\
\text { LLPV: d:ia - } 11\end{array}$ & wasn't operated & $\cdot$ & - & - & $\cdot$ \\
\hline 6 & LUPV/LLPV collector - 12 & $\begin{array}{l}\text { LUPV: d:ia - 33; LUPV: u.ia - 35; } \\
\text { LLPV: d:ia - } 19\end{array}$ & wasn't operated & - & - & $\begin{array}{l}\text { LUPV/LLPV } \\
\text { collector }-4\end{array}$ & - \\
\hline 7 & $\begin{array}{l}\text { PV's collector - left atrium } \\
\text { meangradient - } 11\end{array}$ & $\begin{array}{l}\text { RUPV occlusion } \\
\text { LLPV: dita - } 34 \text { LUPV: uia - } 34 \\
\text { LUPV:d:ia - } 41 \text { LLPV:d:ia - } 30\end{array}$ & wasn't operated & - & - & - & - \\
\hline 8 & RUPV - 6 & $\mathrm{~N}$ cross-sectional area index & RUPV free of stenosis & absence of stenosis & $\mathrm{N}$ cross-sectional area index & $\begin{array}{l}\text { absence of } \\
\text { stenosis }\end{array}$ & $\begin{array}{l}N \text { cross-sectional } \\
\text { area index }\end{array}$ \\
\hline 9 & $\begin{array}{l}\text { Pulmonary hypertension } \\
\text { with undefined cause }\end{array}$ & $\begin{array}{l}\text { RUPV:d:ia - 13; RUPV:u:ia - 29 } \\
\text { RLPV: dia - 14; RLPV: u:ia - } 16 \\
\text { LLPV:dia - } 23 \\
\text { LUPV occlusion }\end{array}$ & $\begin{array}{l}\text { right PVs and LLPV } \\
\text { subocclusion } \\
\text { LUPV atresia }\end{array}$ & $\begin{array}{l}\text { LUPV }-2,5 \\
\text { LLPV }-2,5\end{array}$ & $\begin{array}{l}\text { RUPV:d:ia - 33; RUPV:u:ia -38 } \\
\text { LUPV:d:ia-38 }\end{array}$ & $\begin{array}{l}\text { RUPV - } 4 \\
\text { LUPV-3 }\end{array}$ & $\begin{array}{l}\text { RUPV:d:ia-6 } \\
\text { RUPV:U:ia - } 29 \\
\text { RLPV:d:ia }-35 \\
\text { LUPV:d:ia - } 25 \\
\text { LLP:d:ia - } 11\end{array}$ \\
\hline 10 & left PVs collector stenosis & $\begin{array}{l}\text { left PVs collector:d:ia - } 10 \\
\text { left PVs collector:U:ia - } 16\end{array}$ & $\begin{array}{l}\text { left pVs collector } \\
\text { subocclusion }\end{array}$ & $\begin{array}{l}\text { left PVs collector } \\
\text { stenosis }\end{array}$ & $\begin{array}{l}\text { left PVs collector:d:ia - } 21 \\
\text { left PVs collector:u:ia - } 34\end{array}$ & cied & $\begin{array}{l}\text { left PVs collect:dîa } \\
-21 \\
\text { left PVs collect:u:ia } \\
-34\end{array}$ \\
\hline 11 & LLPV -4 & $\begin{array}{l}\text { LUPV:d:ia - 15; LUPV: U:ia - 23; } \\
\text { LUPV:d:ia - 14; LUPV: u:ia - 43; }\end{array}$ & left PVs local stenosis & absence of stenosis & $\begin{array}{l}\text { RUPV:dia - 11; } \\
\text { LUPV:u:ia - 41; }\end{array}$ & $\begin{array}{l}\text { right PVs } \\
\text { stenosis was } \\
\text { suspected }\end{array}$ & $\begin{array}{l}\text { RUPV:d:ia - } 6 \\
\text { RUPV:U:ia - } 38 \\
\text { LUPV:đ:ia - } 9 \\
\end{array}$ \\
\hline
\end{tabular}

cross-sectional modalities were determined as those providing higher diagnostic accuracy.

\section{Discussion}

We studied diagnostic accuracy of echocardiography for assessment of pulmonary veins characteristics and compared it with CT/MRI method. We found that only in four patients CT/MRI methods completely confirmed echo findings. In some cases echocardiography could not pro- vide visualization of individual pulmonary veins. Subsequently, CT method defined these veins as suboccluded or atretic. Also, in these cases echo was not able to reliably detect the severity of the stenosis: CT/MRI method characterized individual veins as severely stenosed, whereas echocardiography only suspected possible stenosis of the same veins. Likewise, we observed a case where echocardiography showed high mean pressure gradients on an in-
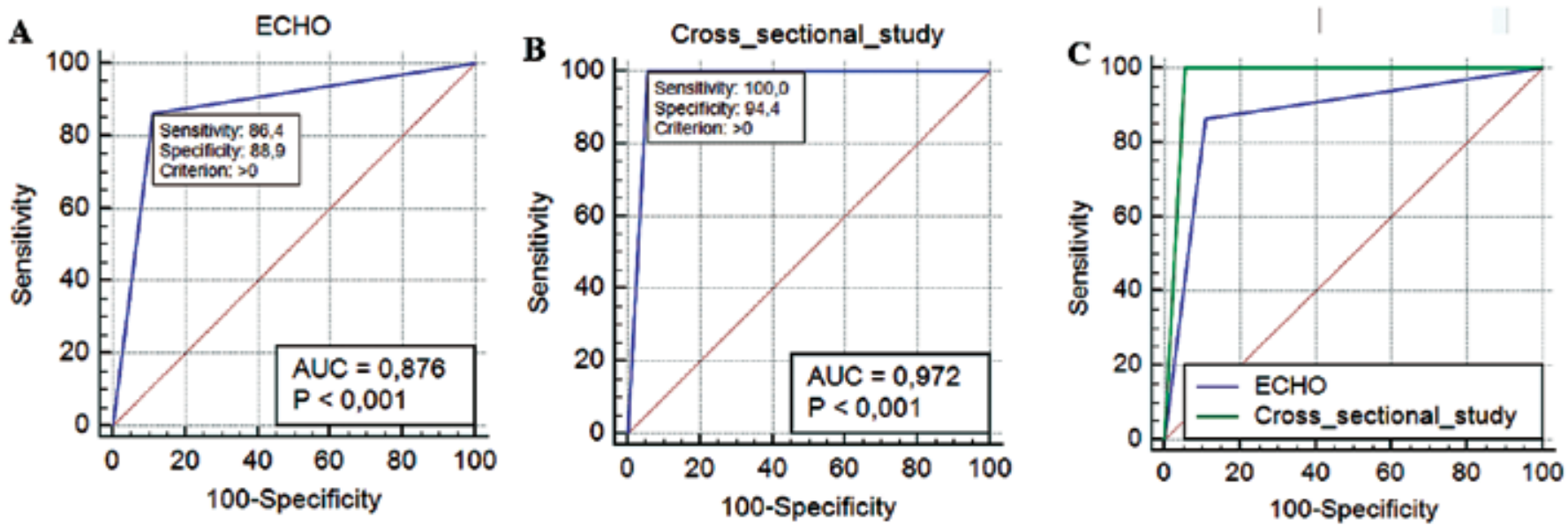

Figure 3. ROC curves of sensitivity and specificity of echo (a) and cross-sectional study (b) with their comparison (c) 
dividual vein. CT method excluded this diagnosis. In a patient with infective endocarditis CT contributed to inform the surgical team of the possible scenario and to plan the surgical procedure, which subsequently was performed with deep hypothermic circulatory arrest. In addition, in case when echocardiography showed severe pulmonary hypertension with normal values of blood flow velocity in pulmonary veins, CT/MRI imaging allowed to determine the cause of detected findings.

Current scoring systems developed to assess the characteristics of pulmonary veins are based mainly on echocardiographic measurements [5]. Echocardiography is mostly accurate in measurement of pulmonary veins at downstream level and when there is no flow redistribution from lung segments with obstructed pulmonary veins to unobstructed segments. In case of unbalanced circulation between both lungs, assessment of pulmonary veins characteristics via echocardiography can lead to underestimation of the stenosis severity [9]. Tremblay et al. demonstrated that echocardiography couldn't provide complete visualization of individual pulmonary veins in the majority of cases [10]. Compared to echo, CT scan can reveal affected pulmonary veins segments that are remote from the heart (upstream).Thus, CT/MRI imaging provides a more comprehensive assessment of anatomy of pulmonary veins compared with traditional echocardiographic imaging. Currently, CT/MRI imaging could be considered as the gold standard for the evaluation of PV flow and anatomy. Vanderlaan et al. [11] adopted a protocol incorporating computed tomography or magnetic resonance imaging at 1,6 , and 12 months after PVS repair. Primary PVS is mostly characterized by abnormal neointimal proliferation, whereas in postsurgical patients heavily collagenized scarring is the most prominent histological finding [12]. These underlying morphology predisposes high recurrence rates and need for rigorous surveillance. Due to its zero radiation load and flow-measurement capabilities MRI may be more beneficial surveillance tool in PVS cohort.

According to our previous approach, CT/MRI imaging was performed only in complex cases [13] mostly to clarify the anatomy. Since April 2018 all patients in our institution with PVS undergo cross-sectional imaging for the verification of diagnosis and surveillance after repair.

\section{Conclusion}

This study recognizes the limitations of echocardiography as an imaging tool for pulmonary veins morphology assessment. Cross-sectional studies overcome these limitations and provide excellent morphological as well as functional (MRI) evaluation of pulmonary veins. We have implemented CT/MRI scans for diagnosis and surveillance in all patients with PVS.

\section{References}

1. Bernstein J, Noke AC, Reed JO. Extrapulmonic stenosis of the pulmonary veins. Circulation. 1959;19(6):891-7. https://doi. org/10.1161/01.cir.19.6.891
2. Kovach AE, Magcalas PM, Ireland C, McEnany K, Oliveira AM, Kieran MW, et al. Paucicellular Fibrointimal Proliferation Characterizes Pediatric Pulmonary Vein Stenosis: Clinicopathologic Analysis of 213 Samples From 97 Patients. Am J Surg Pathol. 2017 Sep;41(9):1198-204. https://doi. org/10.1097/PAS.0000000000000892

3. Caldarone CA, Najm HK, Kadletz M, Smallhorn JF, Freedom RM, Williams WG, et al. Relentless pulmonary vein stenosis after repair of total anomalous pulmonary venous drainage. Ann Thorac Surg. 1998;66(5):1514-20. https://doi. org/10.1016/s0003-4975(98)00952-7

4. Seale AN, Uemura H, Webber SA, Partridge J, Roughton M, Ho SY, et al. Total anomalous pulmonary venous connection: morphology and outcome from an international populationbased study. Circulation. 2010;122(25):2718-26. https://doi. org/10.1161/CIRCULATIONAHA.110.940825

5. Viola N, Alghamdi AA, Perrin DG, Wilson GJ, Coles JG, Caldarone CA. Primary pulmonary vein stenosis: the impact of sutureless repair on survival.

J Thorac Cardiovasc Surg. 2011;142(2):344-50. https://doi. org/10.1016/j.jtcvs.2010.12.004

6. Kalfa D, Belli E, Bacha E, Lambert V, di Carlo D, Kostolny $\mathrm{M}$, et al. Outcomes and Prognostic Factors for Post-Surgical Pulmonary Vein Stenosis in the Current Era. J Thorac Cardiovasc Surg. 2018;156(1):278-86. https://doi.org/10.1016/j. jtcvs.2018.02.038

7. Lo Rito M, Gazzaz T, Wilder TJ, Vanderlaan RD, Van Arsdell GS, Honjo O, et al. Pulmonary vein stenosis: severity and location predict survival after surgical repair. J Thorac Cardiovasc Surg. 2016;151(3):657-66. https://doi.org/10.1016/j. jtcvs.2015.08.121

8. Haycock GB, Schwartz GJ, Wisotsky DH. Geometric method for measuring body surface area: a height-weight formula validated in infants, children, and adults. J Pediatr. 1978;93(1):626. https://doi.org/10.1016/s0022-3476(78)80601-5

9. Kotani Y, Zhu J, Grosse-Wortmann L, Honjo O, Coles JG, Van Arsdell GS, et al. Anatomical risk factors, surgical treatment, and clinical outcomes of left-sided pulmonary vein obstruction in single-ventricle patients. J Thorac Cardiovasc Surg. 2015;149(5):1332-8. https://doi.org/10.1016/j. jtcvs.2014.11.089

10. Tremblay C, Yoo SJ, Mertens L, Seed M, Jacques F, Slorach $\mathrm{C}$, et al. Sutureless Versus Conventional Pulmonary Vein Repair: A Magnetic Resonance Pilot Study. Ann Thorac Surg. 2018 Apr;105(4):1248-54. https://doi.org/10.1016/j.athoracsur.2017.11.015

11. Vanderlaan RD, Caldarone CA. Sutureless repair and postoperative residual stenosis: "Never leave with a lesion". J Thorac Cardiovasc Surg. 2018;156(1):287-8. https://doi.org/10.1016/j.jtcvs.2018.03.080

12. Mykychak Y, Kozhokar D, Yusifli I, Yachnik O, Pavlova A, Zakharova V, Yemets I. Relationship between clinical features and histopathologic findings in patients with pulmonary vein stenosis. Ukrainian journal of cardiovascular surgery. 2019 May 22;(2(35)):61-3. https://doi.org/10.30702/ujcvs/19.3505/044061-063

13. Mykychak Y, Fedevych O, Maksymenko A, Yemets I. Simultaneous arterial switch and totally anomalous pulmonary venous connection repair in a 5-hour-old child, complicated by pulmonary venous stenosis. Interact 
Mykychak Y. / Comparison of Echocardiography and Computed Tomography/Magnetic Resonance Imaging...

Cardiovasc Thorac Surg. 2017;24(5):809-10. https://doi. org/10.1093/icvts/ivw452

14. Kalfa D, Belli E, Bacha E, Lambert V, di Carlo D, Kostolny $\mathrm{M}$, et al. Primary Pulmonary Vein Stenosis: Outcomes,
Risk Factors, and Severity Score in a Multicentric Study. Ann Thorac Surg. 2017;104(1):182-9. https://doi. org/10.1016/j.athoracsur.2017.03.02

\section{Оцінювання морфології легеневих вен у дітей: порівняння методів ехокардіографії та комп'ютерної / магнітно-резонансної томографії}

Микичак Я. Б., лікар-хірург серцево-судинний, відділення серцевої хірургії новонароджених, https://orcid.org/0000-0002-7712-7275

Кожокар Д. М., лікар-інтерн хірург дитячий, відділення серцевої хірургії новонароджених, https://orcid.org/0000-0002-3579-8732

Юсіфлі І. Б., лікар-інтерн хірург дитячий, відділення серцевої хірургії новонароджених, https://orcid.org/0000-0002-6787-6897

Морковкіна А. $є$., лікар-радіолог, відділення радіології

Таммо Раад, канд. мед. наук, лікар-радіолог, відділення радіологіï, https://orcid.org/0000-0001-7506-9449

Ємець І. М., д-р мед. наук, професор, лікар-хірург серцево-судинний, https://orcid.org/0000-0002-1893-0164

ДУ «Науково-практичний медичний центр дитячої кардіології та кардіохірургії МОЗ України», м. Київ, Україна

Резюме. Стеноз легеневих вен - це рідкісне прогресуюче захворювання легеневих вен з високою частотою рецидиву та смертності. Хірургічна корекція не завжди суттєво покращує результати та пов'язана з відносно високими показниками смертності (46-80\%). Основними методами діагностики є ультразвукове дослідження серця та комп'ютерна томографія (КТ) / магнітно-резонансна томографія (МРТ). Візуалізація легеневих вен важлива для передопераційного планування, оцінювання результатів і подальшого спостереження. Різні способи візуалізації можуть надати цінну інформацію про тяжкість і локалізацію уражень. Однак кожен метод має свої переваги та обмеження.

Мета роботи. Метою нашого дослідження є оцінити діагностичну точність ехокардіографії, КТ чи МРТ для визначення характеристик легеневих вен з подальшою розробкою алгоритму виявлення та спостереження за пацієнтами зі стенозом легеневих вен.

Матеріали та методи. Наше дослідження включає 11 пацієнтів, які пройшли комплексне оцінювання анатомії легеневих вен у період з квітня 2018 по червень 2019 року в Центрі дитячої кардіології та кардіохірургії Мо3 України (м. Київ). У всіх пацієнтів було проведено двовимірне та доплерографічне ехокардіографічне дослідження кожної легеневої вени з наступною КТ або МРТ. Усього проведено 27 КТ/МРТ.

Результати. У нашому дослідженні у чотирьох пацієнтів результати ехокардіографії були повністю підтверджені методом КТ/МРТ. Також в окремих пацієнтів важливий стеноз був недооцінений під час ехокардіографії. Чутливість та специфічність ехокардіографії становила 86,4 та 88,9; КТ/МРТ - 100 та 94,4 відповідно.

Висновок. Наше дослідження виявило обмеження ехокардіографії як діагностичого методу для оцінювання морфології легеневих вен. Мультимодальне дослідження забезпечує комплексне морфологічне оцінювання легеневих вен, дозволяє повноцінне планування втручання при стенозі легеневих вен та виявлення рецидивів. Згідно з нашим попереднім підходом, КТ/МРТ проводили лише у складних випадках, переважно для уточнення анатомії. У нашому дослідженні ми визнали обмеження ехокардіографії як інструменту візуалізації для оцінювання морфології легеневих вен. Як результат, з квітня 2018 року всі пацієнти в нашому закладі зі стенозом легеневих вен проходять КТ/МРТ для уточнення діагнозу та подальшого спостереження після корекції.

Ключові слова: стеноз легеневих вен, ехокардіографія, комп'ютерна томографія, магнітно-резонансна томографія.

Стаття надійшла в редакцію 14.05.2020 р. 